\title{
Clinical Trial Dose Escalation Document
}

National Cancer Institute

\section{Source}

National Cancer Institute. Clinical Trial Dose Escalation Document. NCI Thesaurus. Code C115647.

Records detailing the correspondence and decisions regarding dose escalation during a clinical trial. 